\title{
ReCurRent Gestational Hypertriglyceridemia InduCEd Pancreatitis in Pregnancy
}

TAN Ilka ${ }^{1}$, JUNG Jacqueline ${ }^{2}$, TAN Toh Lick ${ }^{2,3}$

${ }^{1}$ Department of Maternal and Fetal Medicine, ${ }^{2}$ Department of Obstetrics and Gynaecology, KK Women's and Children's Hospital, Singapore

${ }^{3}$ Obstetrics and Gynaecology, Thomson Women's Clinic (Jurong East), Thomson Wellth Clinic, Singapore

\section{Introduction}

Gestational hypertriglyceridemia is a rare but potentially serious condition in pregnancy. It can lead to acute pancreatitis with high maternal and fetal morbidity and mortality. It is associated with preterm delivery and has a high recurrence rate in subsequent pregnancies. We present a case report and literature review of recurrent gestational hypertriglyceridemia leading to acute pancreatitis in pregnancy.

\section{Case report}

Madam A is a 26 year old Para 1 with a previous emergency caesarean section at 34 weeks for acute pancreatitis due to gestational hypertriglyceridemia. In her second pregnancy, she was booked at another hospital and transferred her care to our institution at 30 weeks gestation. She was admitted at $33^{+2}$ weeks with acute pancreatitis due to gestational hypertriglyceridemia (figure 1). Following her emergency caesarean section her recovery was uncomplicated. Her triglycerides returned to almost normal levels at her 2 months follow up.

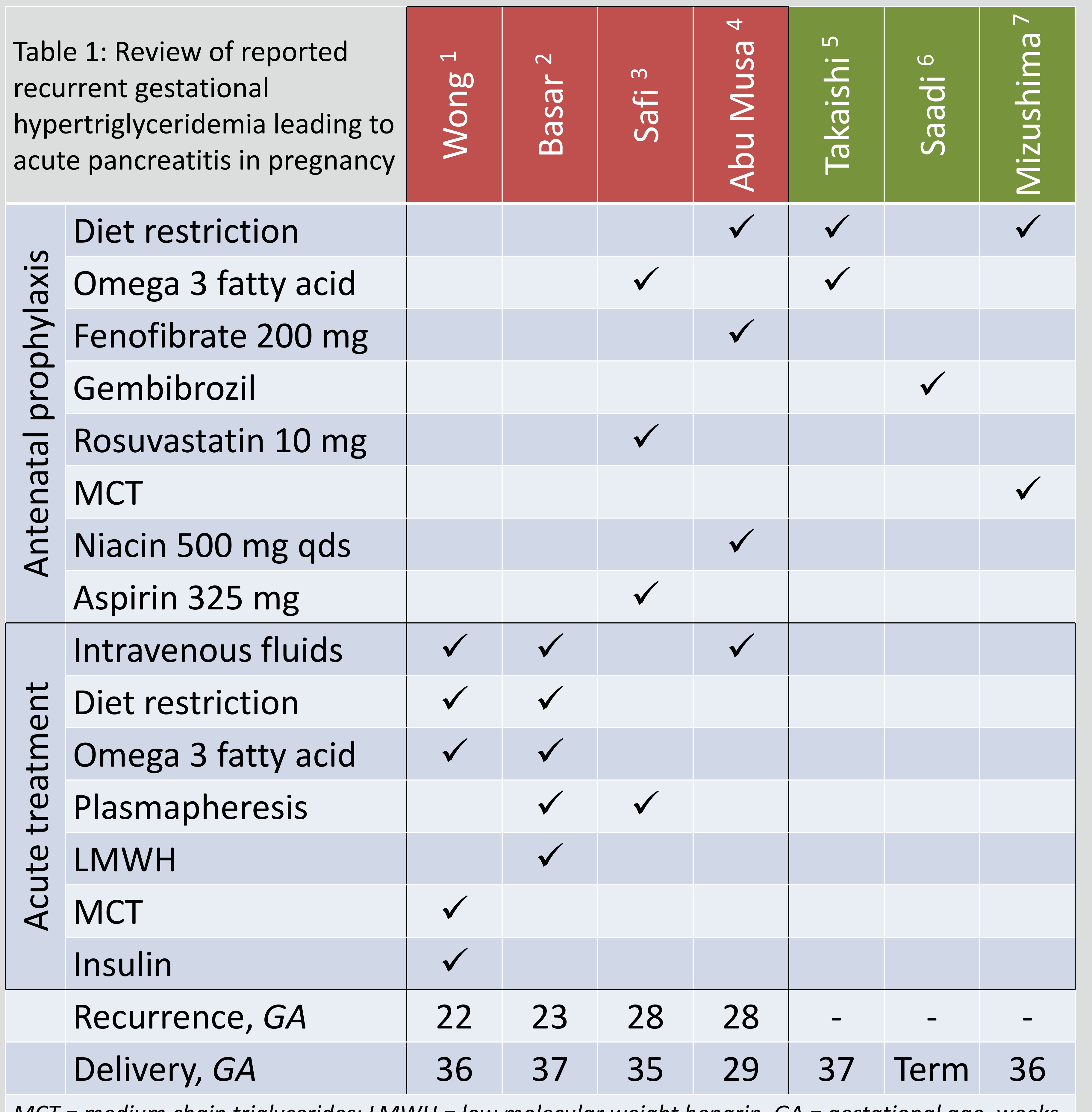

\section{Literature review}

Our literature search identified 7 cases (Table 1). In women who did not have antenatal prophylactic treatment, all had recurrence $(n=2)$. This risk appears to be dramatically reduced to 2 in 5 if they had antenatal prophylactic treatment. Of those with recurrence, early diagnosis and aggressive acute treatment appears to increase recurrence to delivery interval.

\section{Conclusion}

Pregnant women with history of gestational hypertriglyceridemia induced acute pancreatitis may benefit from the following:

- Pre-conception counselling and institution of non-pharmacologic intervention such as diet and exercise

- Multidisciplinary team management including dietician to counsel and encourage a fat restricted diet

- Consider the use of lipid lowering agents such as fibrates and omega- 3 fatty acids after the first trimester

- Regular monitoring of lipid levels in pregnancy particularly in the third trimester. Aim for triglycerides $<500 \mathrm{mg} / \mathrm{dl}$

- Consider hospital admission in the third trimester for further intensification of dietary fat restriction

- When triglycerides rise $>1000 \mathrm{mg} / \mathrm{dl}$, consider fasting with parental nutrition and plasmapheresis

All these interventions aim to avoid recurrence of acute pancreatitis and to prolong pregnancy to minimize premature delivery. Further research is needed to determine the optimal treatment of this rare but important pregnancy induced condition.

References

Severe gestational hypertriglyceridemia: A practical approach for clinicians. Wong B et al. Obstet Med. 2015 Dec; 8(4):158-67.

2. Therapeutic apheresis for severe hypertriglyceridemia in pregnancy. Basar $R$ et al. Arch Gynecol Obstet. 2013 May;287(5):839-43.

3. Management of familial hypertriglyceridemia-induced pancreatitis during pregnancy with therapeutic plasma exchange: a case report and review of literature. Safi F et al.. Am J Ther. 2014 Sep-Oct;21(5):e134-6.

4. Recurrent hypertriglyceridemia-induced pancreatitis in pregnancy: a management dilemma. Abu Musa AA et al. Pancreas. 2006 Mar;32(2):227-8. 5. Hypertriglyceridemic acute pancreatitis during pregnancy: prevention with diet therapy and omega-3 fatty acids in the following pregnancy. Takaishi $K$ et al. Nutrition. 2009 Nov-Dec;25(11-12):1094-7

6. Severe hypertriglyceridemia and acute pancreatitis during pregnancy: treatment with gemfibrozil. Saadi HF et al. Endocr Pract. 1999 JanFeb;5(1):33-6.

7. Prevention of hyperlipidemic acute pancreatitis during pregnancy with medium-chain triglyceride nutritional support. Mizushima T et al. Int J Pancreatol. 1998 Jun;23(3):187-92.

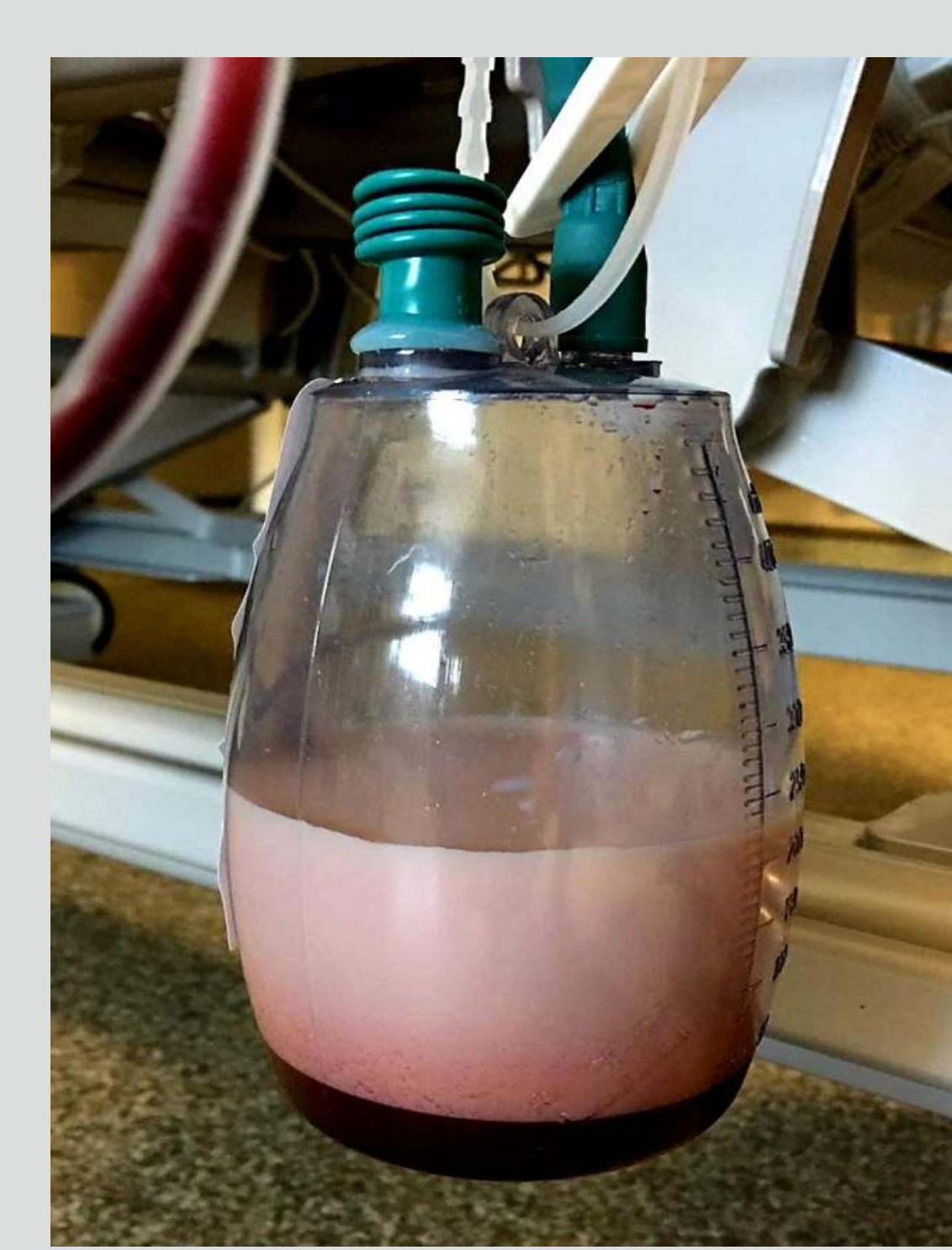

Figure 1: Pink lipaemic peritoneal fluid in our case study with acute pancreatitis complicating gestational hypertriglyceridemia 